\section{Change of foveal and perifoveal thickness measured by OCT after phacoemulsification and IOL implantation}

Z Biro, Z Balla and B Kovacs
Department of

Ophthalmology, University of Pecs, Faculty of Medicine, Pécs, Baranya, Hungary

Correspondence: Z Biro, Department of

Ophthalmology,

University of Pecs,

Faculty of Medicine, H-7643. Pecs, Ifjusag Street 13, Pecs, Baranya 1623 Hungary

Tel: + 3672536141

Fax: + 3672536142

E-mail: zsolt.biro@aok.pte.hu

Received: 25 September 2005

Accepted in revised form: 25 April 2006

Published online: 2 June 2006

\begin{abstract}
Purpose Cataract surgery is known to elicit postoperative macular oedema owing to its traumatic effect. The aim of the study was to measure the foveal and perifoveal thickness of the retina after uneventful phacoemulsification and posterior chamber intraocular lens (PC-IOL) implantation.

Methods Retinal thickness values of the foveal and perifoveal (fovea $+3.0 \mathrm{~mm}$, fovea $+6.0 \mathrm{~mm}$ ) sectors and the minimum values were measured in a prospective study using optical coherence tomography (OCT) in 71 eyes of 71 patients (34 men, 37 women, mean age: 68.8 years) who underwent cataract surgery. Phacoemulsification and 'in the bag' implantation of a foldable PC-IOL was performed under topical anaesthesia. Data were collected 1 day before the operation, and postoperatively at $1,7,30$, and 60 days. Contralateral eyes of each patient served as controls. 'Student's $t$-test' was used for statistical analysis, and $P<0.05$ value was considered significant.

Results No significant change of the thickness values could be measured 1 day after surgery. However, a significant increase could be detected on the postoperative 7,30 , and 60 days in the perifoveal 3.0 and $6.0 \mathrm{~mm}$ sectors either calculated alone or averaged together with the foveal values. The initial (preoperative) average value of $234.1 \pm 2.6 \mu \mathrm{m}$ (mean \pm SEM, $n=536$ ) in the $6.0 \mathrm{~mm}$ perifoveal region increased to $242.5 \pm 2.6 \mu \mathrm{m}$ (mean \pm SEM, $n=488, P<0.01$ ) 1 week, to $247.7 \pm 4.6 \mu \mathrm{m}(n=352, P<0.01) 1 \mathrm{month}$, and to $246.0 \pm 5.9 \mu \mathrm{m}(n=208, P<0.05) 2$ months after surgery, which proved to be significant.
\end{abstract}

However, the relative change of macular thickness was moderate, 3.5, 5.6, and 5.3\% at the above periods, respectively.

In a subgroup of patients $(n=34)$ minimum 6 months after cataract surgery, the $6.0 \mathrm{~mm}$ perifoveal values were found to be elevated at $(237.8 \pm 3.9 \mu \mathrm{m}$, mean \pm SEM, $n=272)$ as compared to the control eyes $(233.9 \pm 3.8 \mu \mathrm{m}$, mean \pm SEM, $n=272$ ). The difference was $1.7 \%$ and proved not to be significant $(P>0.05)$. Conclusions Mild subclinical perifoveal oedema following cataract surgery was detected using OCT at postoperative day 7 to 6 months. The minimal and the foveal values in itself are inadequate to describe the phenomenon. The macular thickness values of the perifoveal 3.0 and $6.0 \mathrm{~mm}$ sectors alone or averaged together with the foveal values provide more accurate description of the postoperative macular oedema.

OCT enables the detection of minimal increases in perifoveal retinal thickness even 6 months after cataract surgery.

Eye (2008) 22, 8-12; doi:10.1038/sj.eye.6702460; published online 2 June 2006

Keywords: macular oedema; optical coherence tomography; perifoveal oedema; phacoemulsification

Introduction

It is well known that macular oedema may develop after intraocular surgery. Postoperative macular oedema was recognised frequently after intracapsular cataract extraction (ICCE), especially in cases of vitreous loss, which has 
been named Irvine-Gass syndrome after the discoverers. ${ }^{1,2}$ Although the exact pathomechanism is not known, the role of surgical trauma and release of prostaglandin, which may increase the permeability of the perifoveal capillaries, ${ }^{3}$ is suspected. Not only vitreoretinal traction but also postoperative hypotony may play a role in the pathogenesis. Macular oedema may severely decrease the postoperative visual acuity (VA). With ICCE, the rate of postoperative macular oedema was around 2-20\%, whereas it decreased to 1-2\% with the widespread use of extracapsular cataract extraction (ECCE) with intact posterior capsule. With the introduction of phacoemulsification, the rate of postoperative macular oedema is even less. ${ }^{4-6}$

The aim of this prospective study was to examine the change of the foveal and perifoveal retinal thickness in a relatively large number of patients $(n=71)$ with OCT (optical coherence tomography) in the first 60 days after uneventful phacoemulsification and foldable posterior chamber intraocular lens (PC-IOL) implantation.

OCT is an objective, noninvasive, well-tolerated, 'noncontact' method for quantitative measurements, with good reproducibility and with $10 \mu \mathrm{m}$ resolution. ${ }^{7}$

\section{Patients and methods}

In a prospective study, 71 eyes of 71 patients (34 men, 37 women, average age 68.8 years, SD 8.8 years) were examined by OCT. The retinal thickness in the fovea and perifoveal areas (fovea $+3.0 \mathrm{~mm}$, fovea $+6.0 \mathrm{~mm}$ ) was measured on the preoperative day, and on postoperative days $1,7,30$, and 60 . During OCT examinations, we performed six scans in $6 \mathrm{~mm}$ length centred on the fovea. Cataract surgeries were performed under topical anaesthesia, using a standard technique. After the clear corneal incision, capsulorhexis was made with forceps under Amvisc Plus (sodium hyaluronate), and a 'divide and conquer' phacoemulsification technique was used. PC-IOL was implanted 'in the bag' in all cases, and then the viscoelastic material was carefully washed out from the anterior chamber and from behind the PC-IOL using the 'two-compartment technique' of Tetz and Holzer. ${ }^{8}$ All the cases were senile cataract from grade I to III. The time of phacoemulsification varied between 21 and $171 \mathrm{~s}$, and the mean phacoenergy was between 40 and $50 \%$.

Exclusion criteria were previous intraocular surgery and any intraoperative or postoperative complication. The perioperative and the postoperative treatment was the same in all patients.

Pupils were dilated for OCT examination in all cases with $1 \%$ tropicamide (Mydrum) or $2.5 \%$ phenylephrine and measurements were performed on the fellow eyes as well, as controls. 'Student's $t$-test' was used for statistical analysis, and $P<0.05$ value was considered significant.
In a smaller group of patients (34 eyes), the retinal thickness in the fovea and the 3.0 and $6.0 \mathrm{~mm}$ perifoveal area was measured minimum 6 months (average 8.6 months) after cataract surgery, and the results were compared to the nonoperated fellow eye.

\section{Results}

No significant change in the foveal and perifoveal retinal thickness values was found on the first postoperative day, compared to the preoperative values.

On the other hand, there was a significant increase of the perifoveal 3.0 and $6.0 \mathrm{~mm}$ diameter retinal area thickness either examined alone or together with the foveal area thickness on day 7,30, and 60 postoperatively (Figure 1). The preoperative value was $234.1 \pm 2.6 \mu \mathrm{m}$ (average $\pm \mathrm{SEM}, n=536$ ) on the $6.0 \mathrm{~mm}$ area. After 1 week this value increased to $242.5 \pm 2.6 \mu \mathrm{m}$ (average $\pm \mathrm{SEM}, n=488$ ), after 1 month it increased to $247.7 \pm 4.6 \mu \mathrm{m}$ (average $\pm \mathrm{SEM}, n=352$ ), and after 2 months it increased to $246.0 \pm 5.9 \mu \mathrm{m}$ (average $\pm S E M$, $n=208)$, which proved to be significant $(P<0.05)$ (Figure 2). The change of the $6.0 \mathrm{~mm}$ diameter perifoveal area is not very high in per cent, after 1 week it was 3.5\%, after 1 month $5.6 \%$, and after 2 months it was $5.3 \%$.

The postoperative VA of all of the patients was 0.5 or better already on the first postoperative day, which improved to $0.86(0.6-1.0)$ on the average by the end of the second month postoperatively.

In a retrospective study, a smaller group of patients (34 eyes) underwent uncomplicated cataract surgery minimum 6 months before, increased values of the $6.0 \mathrm{~mm}$ diameter perifoveal area were measured: $237.8 \pm 3.9 \mu \mathrm{m}$, (average \pm SEM, $n=272$ ) compared to the average value of the nonoperated fellow eyes: $233.9 \pm 3.8 \mu \mathrm{m}$ (average $\pm \mathrm{SEM}, n=272$ ). The difference was $1.7 \%$ and proved to be not significant $(P>0.05)$ (Figure 3 and Table 1).

\section{Discussion}

Increase of the foveal and perifoveal retinal thickness after uncomplicated cataract surgery was demonstrated by OCT in this study on postoperative 7,30 , and 60 th days.

OCT is a new diagnostic tool, which gives a highresolution cross-sectional image about the change of the retina and chorioidea in the living structure (in vivo). The eye is illuminated with a near-infrared light, the technique is noninvasive. Since it is a so-called 'noncontact' method, it is well tolerated by the patients. Dilation of the pupil helps to perform the examination, but it is not absolutely necessary. Reproducibility of the method is very good, and its accuracy is around $10 \mu \mathrm{m} .{ }^{9}$ 

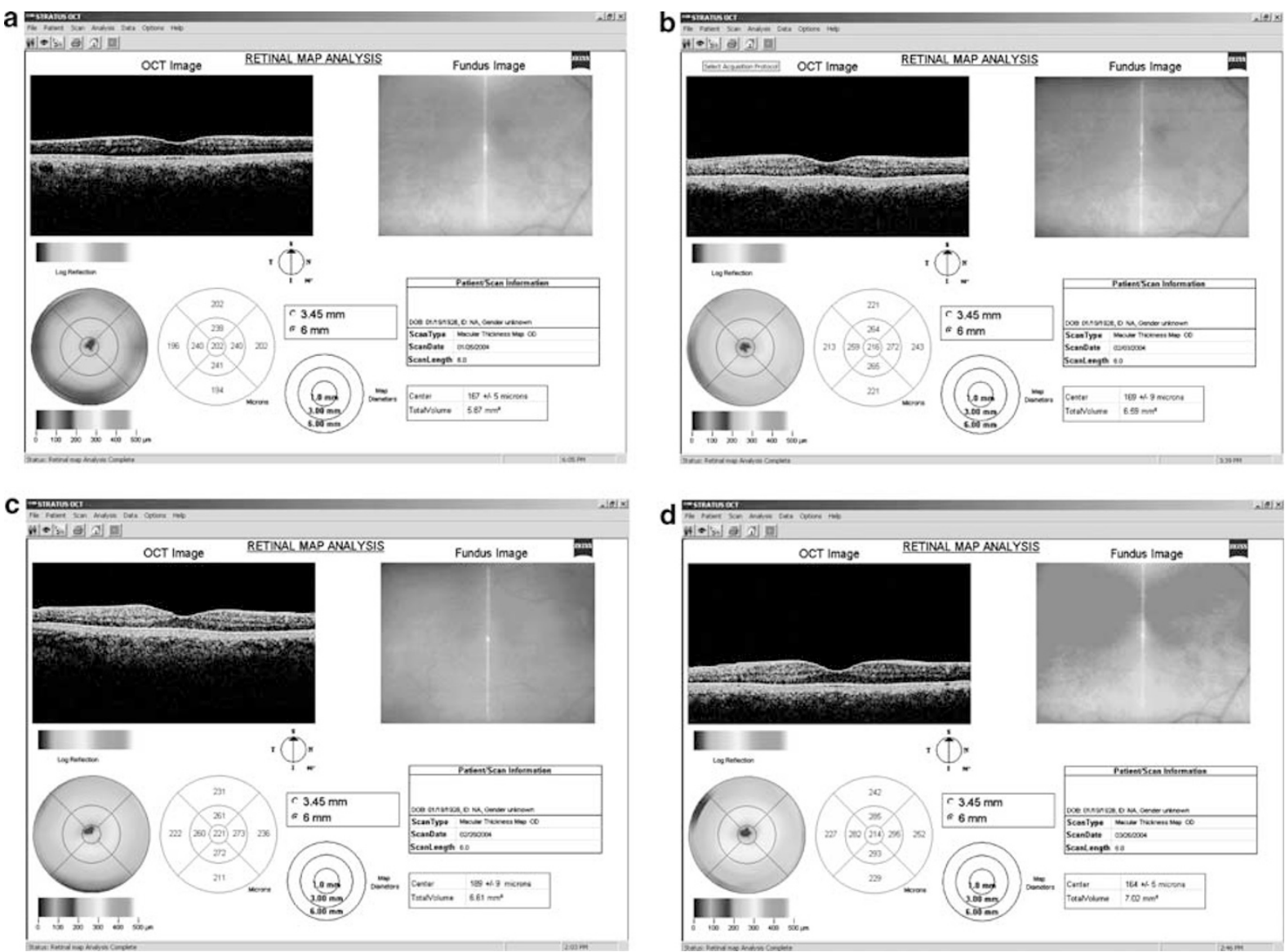

Figure 1 OCT pictures of the fovea and the perifoveal 3.0 and $6.0 \mathrm{~mm}$ diameter area. (a) Before surgery, (b) 7 postoperative days, (c) 30 postoperative days, and (d) 60 postoperative days. The average values of the retinal thickness of the different areas can be seen in the picture $(\mu \mathrm{m})$.
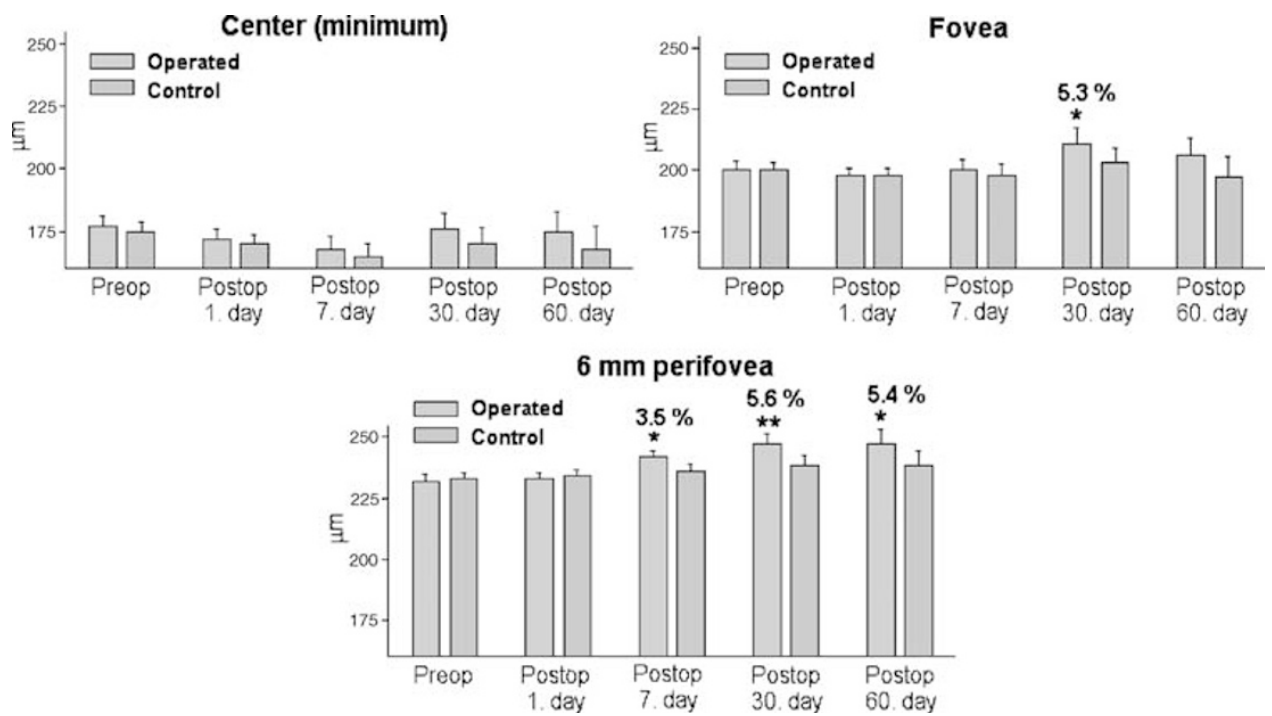

Figure 2 Change of retinal thickness in the fovea and in the $6.0 \mathrm{~mm}$ diameter perifoveal area in the 7-60 postoperative days. Values seen in the text $(n=71)$. 'Student's $t$-test' significant, ${ }^{*} P<0.05$, ${ }^{* *} P<0.01$ 
It is mainly useful in the examination of the changes of the anatomical structures of the macular area (oedema, chorioideal neovascularisation, a retinal pigmentepithel detachment, detachment of the neurosensory retina), and in the examination of the disorders of the vitreoretinal surface (such as vitreomacular traction, epiretinal membrane). ${ }^{7,10}$

The results of ophthalmoscopy, fluorescein angiography, and ultrasound examination is supplemented by the possibility of the anatomic examination of the retina, which may facilitate diagnosis and therapy. OCT is a quantitative measurement and the changes in the parameters may allow us to quantitatively assess progression of disease and success of therapy., ${ }^{9,10}$

In another study, the change of retinal thickness was measured in 15 different points of the posterior part of the retina, half an hour after cataract extraction in a small number of patients (10 eyes), and there was no significant change $(P=0.8)$ compared to the preoperative values. ${ }^{11}$

Chinese authors performed OCT examinations 1 week after uneventful cataract surgery on 80 eyes. The average preoperative foveal thickness was measured as

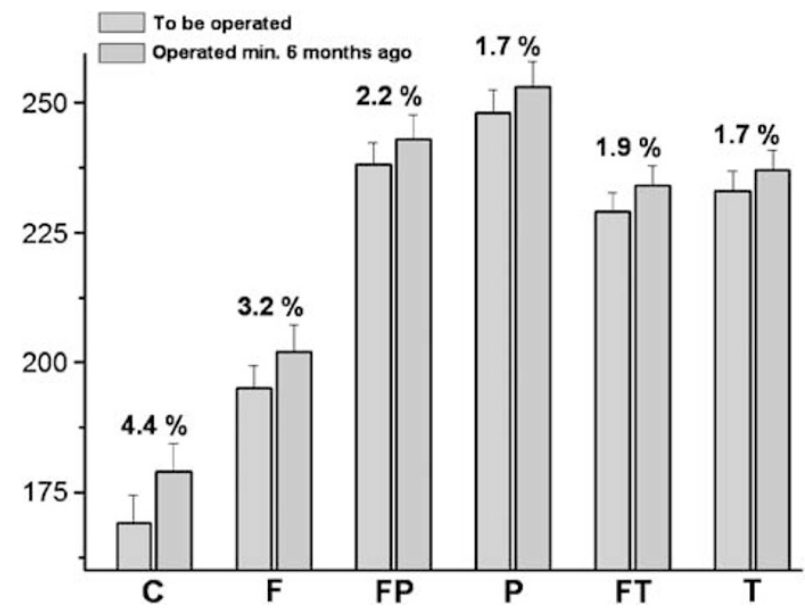

Figure 3 Thickness values of the perifoveal $6.0 \mathrm{~mm}$ diameter area compared to the values of the control (not operated) eyes minimum 6 months after uncomplicated phacoemulsification + PC-IOL implantation $(n=34)$
$142.9 \pm 16.7 \mu \mathrm{m}$, which increased to $157.9 \pm 36.7 \mu \mathrm{m}$ after 1 week and the difference proved to be nonsignificant $(P>0.05)$. On the other hand, they proved that there was a significant correlation $(P<0.05)$ between the amount of phacoenergy used and the postoperative macular thickness. ${ }^{12}$

French authors found macular thickening and decreased VA in 11 eyes out of 41 after uncomplicated cataract extraction. ${ }^{13}$ Postoperative macular oedema is explained by the damage of the blood-retinal barrier. ${ }^{14,15}$ On the other hand, no correlation was found between the inflammatory signs in the anterior chamber ('flare') and macular thickness. According to their opinion, damage of the blood-retinal barrier and consequent thickening of the macular area may happen without the damage of the blood aqueous barrier.

OCT examinations were performed to measure the thickness of the retinal nerve fibre layer after filtration glaucoma surgery on 38 eyes. ${ }^{16}$ A major increase in the retinal nerve fibre thickness $(P<0.0001)$ was found as the IOP decreased from 22.0 to $11.4 \mathrm{mmHg}$. Although the exact pathomechanism is not known, it is assumed that the lamina cribrosa moves forward as the consequence of decrease of the IOP and so the nerve fibre layer may regain its original shape and thickness, until the glaucomatous changes are not irreversible.

Although it is known that the length of phacoemulsification surgery and the phacotime as well as the phacoenergy all may influence the outcome, these parameters were not evaluated in our study. An average $40-50 \%$ phacoenergy was used in all of the cases.

As the postoperative VA of all of the patients was 0.5 or better already on the first postoperative day, postoperative VA and OCT findings were not correlated in this study.

\section{Conclusions}

Increase in the retinal thickness of the perifoveal $6.0 \mathrm{~mm}$ diameter area can be demonstrated by OCT in the 7-60 days postoperative period after uncomplicated

Table 1

\begin{tabular}{|c|c|c|c|c|c|c|c|c|c|c|c|c|}
\hline & \multicolumn{6}{|c|}{ Control eye } & \multicolumn{6}{|c|}{ Surgery minimum 6 months ago } \\
\hline & C & $F$ & $F P$ & $P$ & $F T$ & $T$ & C & $F$ & $F P$ & $P$ & $F T$ & $T$ \\
\hline Average & 169.13 & 195.19 & 237.70 & 248.33 & 229.28 & 233.54 & 177.15 & 201.81 & 243.07 & 252.76 & 233.80 & 237.53 \\
\hline SEM & 5.62 & 4.40 & 4.36 & 4.64 & 3.91 & 4.00 & 5.55 & 5.36 & 4.76 & 5.04 & 4.04 & 4.09 \\
\hline$\%$ & & & & & & & 4.74 & 3.39 & 2.26 & 1.78 & 1.97 & 1.71 \\
\hline $\mathrm{N}$ & 27 & 27 & 135 & 108 & 243 & 216 & 27 & 27 & 135.00 & 108 & 243 & 216 \\
\hline $\mathrm{T}$ & & & & & & & 0.22 & 0.06 & 0.07 & 0.14 & 0.06 & 0.10 \\
\hline
\end{tabular}

$\mathrm{C}$, centre (minimum value); $\mathrm{F}$, fovea; FP, fovea $+3 \mathrm{~mm}$ perifoveal area; FT, fovea $+6 \mathrm{~mm}$ perifoveal area; $\mathrm{P}, 3 \mathrm{~mm}$ perifovea; $\mathrm{T}, 6 \mathrm{~mm}$ perifovea. 
phacoemulsification and IOL implantation. The minimum values measured in the fovea by themselves are not suitable to evaluate the postoperative macular oedema. Changes of the perifoveal 3.0 or $6.0 \mathrm{~mm}$ diameter areas themselves or measured together with the foveal (minimum) values are much more suitable to characterise the postoperative macular oedema.

Modern microsurgical techniques and the new generation of anti-inflammatory drugs dramatically decreased the rate of postoperative macular oedema, causing clinical symptoms. Despite these facts, with the help of the objective, noninvasive, good reproducible OCT measurements, the anatomical changes of the retina in the postoperative period can be well followed. Owing to its high sensitivity, OCT is suitable to demonstrate the minimal increase of the perifoveal retinal thickness even 6 months after surgery.

\section{References}

1 Gass JDM, Norton EWD. Cystoid macular edema and papilledema following cataract extraction: a fluorescein funduscopic and angiographic study. Arch Ophthalmol 1966; 76: 646-661.

2 Irvine SR. A newly defined vitreous syndrome following cataract surgery, interpreted according to recent concepts of the vitreous: Francis I.Proctor lecture. Am J Ophthalmol 1953; 36: 599-619.

3 Kosaka T. The effects of prostaglandins on the blood retinal barrier. Nippon Ganka Gakkai Zasshi 1995; 99: 412-419.

4 Kaiya T. Observation of blood-aqueous barrier function after posterior chamber intraocular lens implantation. J Cataract Refract Surg 1990; 16: 320-325.

5 Kraff MC, Sanders DR, Jampol LM, Lieberman HL. Effect of primary capsulotomy with extracapsular surgery on the incidence of pseudophakic cystoid macular edema. Am J Ophthalmol 1984; 98: 166-170.
6 Norregaard JC, Bernth-Petersen P, Bellan L, Alonso J, Black C, Dunn E et al. Intraoperative clinical practice and risk of early complications after cataract extraction in the United States, Canada, Denmark and Spain. Ophthalmology 1999; 106: $42-48$.

7 Hee MR, Izatt JA, Swanson EA, Huang D, Schuman JS, Lin $\mathrm{CP}$ et al. Optical coherence tomography of the human retina. Arch Ophthalmol 1995; 113: 325-332.

8 Tetz MR, Holzer MP. Two compartment technique to remove ophthalmic viscosurgical devices. J Cataract Refract Surg 2000; 26: 641-643.

9 Voo I, Mavrofrides EC, Puliafito CA. Clinical applications of optical coherence tomography for the diagnosis and management of macular diseases. Ophthalmol Clin N Am 2004; 17(1): 21-31.

10 Puliafito CA, Hee MR, Lin CP, Reichel E, Schuman JS, Duker JS. Imaging of macular diseases with optical coherence tomography. Ophthalmology 1995; 102: 217-229.

11 Grewing R, Besker H. Retinal thickness immediately after cataract surgery measured by optical coherence tomography. Ophthalmic Surg Lasers 2000; 31(3): 215-217.

12 Cheng B, Liu Y, Liu X, Ge J, Ling Y, Zheng X. Macular image changes of optical coherence tomography after phacoemulsification. Zhonghua Yan Ke Za Zhi 2002; 38(5): 265-267.

13 Sourdille P, Santiago PY. Optical coherence tomography of macular thickness after cataract surgery. J Cataract Refract Surg 1999; 25(2): 256-261.

14 Konari K, Sawada Z, Zhong Y, Isomura H, Nakagawa T, Mori M. Development of the blood-retinal barrier in vitro: formation of tight junctions as revealed by occludin and ZO-1 correlates with the barrier function of chick retinal pigment epithelial cells. Exp Eye Res 1995; 61: 99-108.

15 Vinores SA, Amin A, Derevjanik NL, Green WR, Campochiaro PA. Immunohistochemical localisation of blood-retinal breakdown sites associated with post-surgical macular edema. Histochem J 1994; 26: 655-665.

16 Aydin A, Wollstein G, Price LL, Fujimoto JG, Schuman JS. Optical coherence tomography assessment of retinal nerve fiber layer thickness changes after glaucoma surgery. Ophthalmology 2003; 110(8): 1506-1511. 